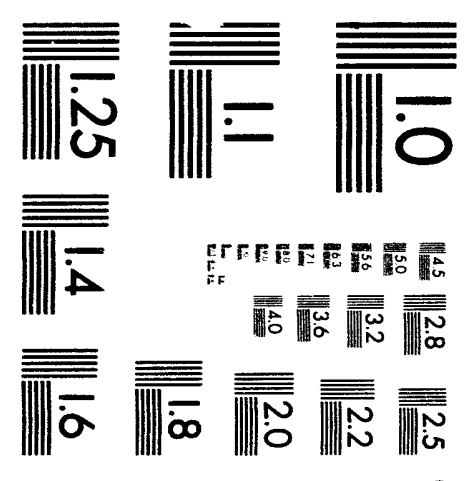



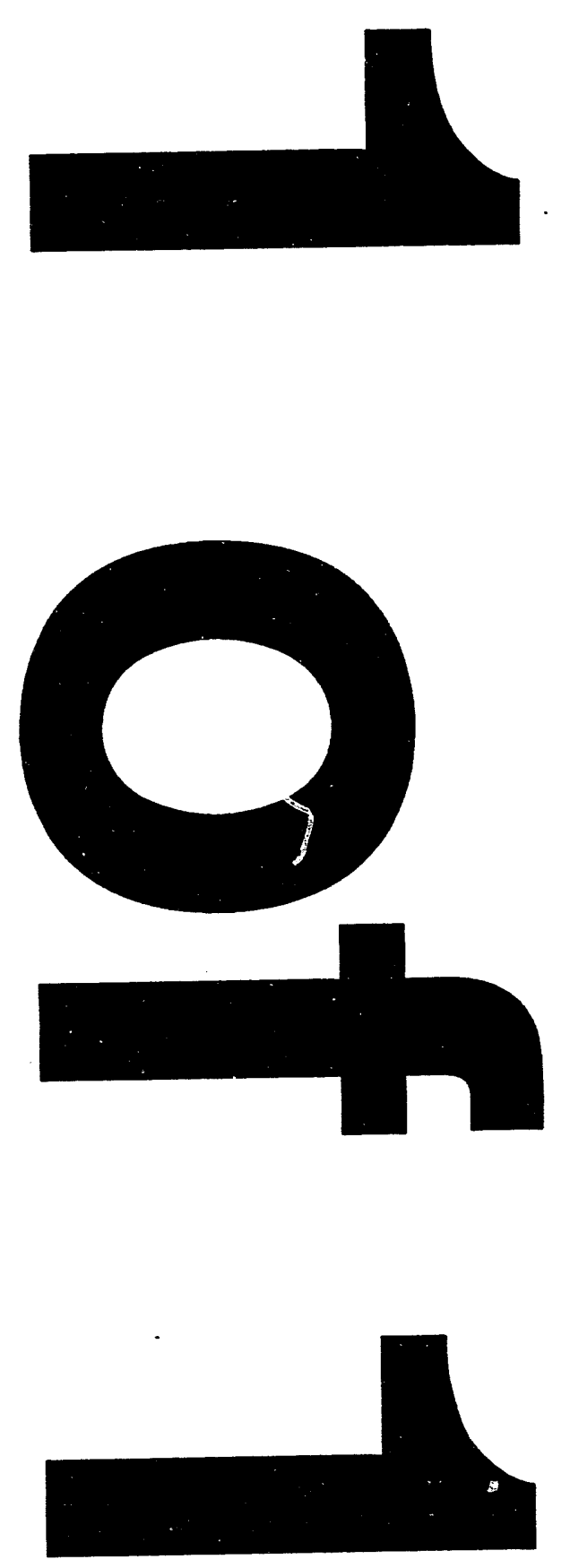


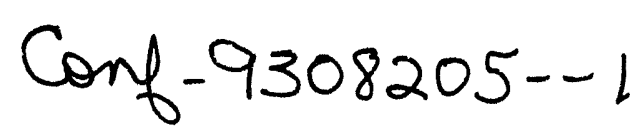

LA-UR-9 3-4251

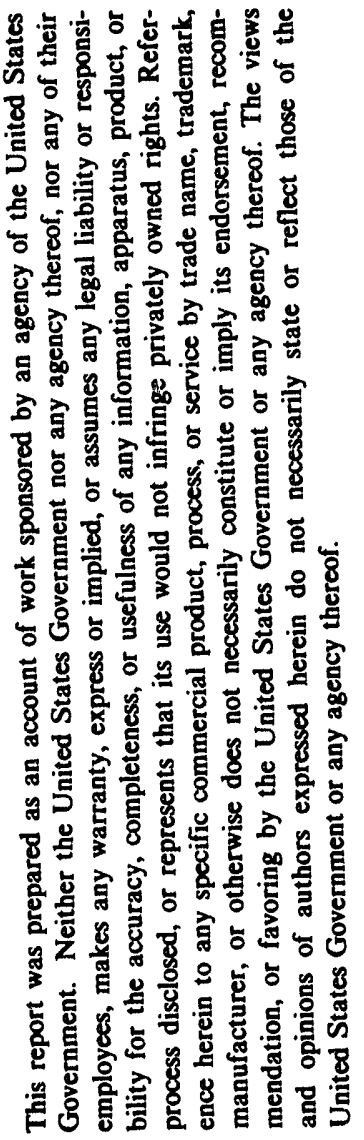

\section{Submitted to:}

K. M. Hanson

D. R. Wolf

Proc. 13th MAXENT WORKSHOP, SANTA BARBARA, 1993

EDITOR: Dr. Glenn Heidbreder

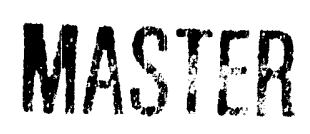

DISTRIBUTION OF THIS DOCUMENT IS UNLIMITED $\rho^{\rho}$

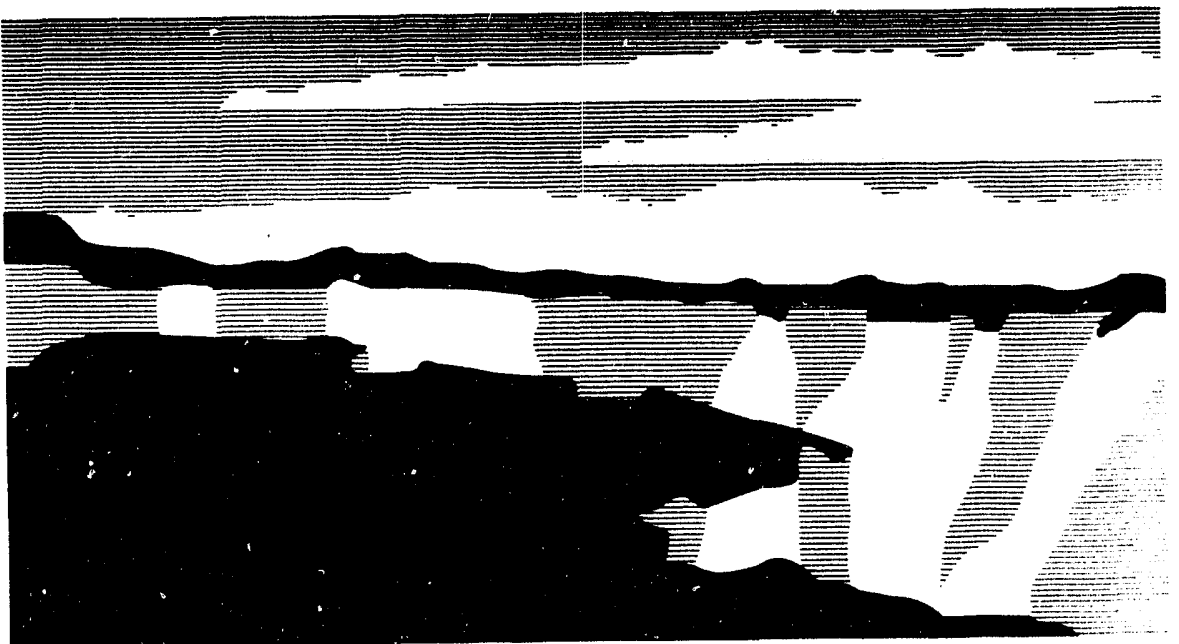

ESTIMATORS FOR THE CAUCHY DISTRIBUTION 


\title{
ESTIMATORS FOR THE CAUCHY DISTRIBUTION
}

\author{
K. M. Hanson and D. R. Wolf \\ Los Alamos National Laboratory, MS P940 \\ Los Alamos, New Mexico 87545 USA \\ email: kmh@lanl.gov and wolf@lanl.gov
}

\begin{abstract}
We discuss the properties of various estimators of the central position of the Cauchy distribution. The performance of these estimators is evaluated for a set of simulated experiments. Estimators based on the maximum and mean of the posterior probability density function are empirically found to be well behaved when more than two measurements are available. On the contrary, because of the infinite variance of the Cauchy distribution, the average of the measured positions is an extremely poor estimator of the location of the source. However, the median of the measured positions is well behaved. The rms errors for the various estimators are compared to the Fisher-Cramer-Rao lower bound. We find that the square root of the variance of the posterior density function is predictive of the rms error in the mean posterior estimator.
\end{abstract}

\section{Introduction}

We explore the properties of various estimators of the central position of the Cauchy distribution, which is notorious for the divergent nature of its first and higher moments. The results of using different kinds of estimators are evaluated by simulating a series of experiments using a Monte Carlo procedure. Investigation of the Cauchy distribution is profitable because its peculiar properties illustrate some interesting aspects of parameter estimation based on Bayesian analysis. It provides us with an example of how to properly deal with data outliers. Some aspects of this paper have been presented in [1].

\section{The Cauchy Distribution}

\subsection{The problem}

Suppose that a radioactive source, located at the position $\left(x_{0}, y_{0}\right)$, emits gamma rays. A position-sensitive linear detector, colinear with the $x$ axis and extending to infinity in both directions, measures the position $x_{i}$ that the $i$ th gamma ray hits the detector. The data consist of the values $x_{i}, i=1, \ldots, N$, which we designate by the vector $\mathbf{x}$. The problem is to estimate the location of the source $x_{0}$, assuming that $y_{0}$ is known. This problem is Gull's lighthouse example [2] cast in another setting.

Assume that the gamma rays are confined to the $x-y$ plane and are emitted uniformly in the angle $\theta$ at which they leave the source. From the relation $\tan (\theta)=-y_{0} /\left(x_{i}-x_{0}\right)$, which holds for $-\pi<\theta<0$, the probability density function of a measurement $x_{i}$ is obtained by using the Jacobian determinant to transform the density function dependence from $\theta$ to $x_{i}$

$$
p\left(x_{i} \mid x_{0}, y_{0}\right)=\frac{y_{0}}{\pi\left[y_{0}^{2}+\left(x_{0}-x_{i}\right)^{2}\right]} .
$$


We call $p\left(x_{i} \mid x_{0}, y_{0}\right)$ the likelihood. It is a properly normalized Cauchy distribution, which is notorious for having an undefined mean and an infinite variance. The width of this distribution may be characterized by its FWHM, which is $2 y_{0}$.

In a Bayesian analysis the posterior probability density function for the $x_{0}$ position of the source summarizes the state of knowledge concerning $x_{0}$ by providing the probability density of every possible value of $x_{0}$. The posterior of $x_{0}$, given the data $\mathbf{x}$ and the position parameter $y_{0}$, is given by Bayes's law

$$
p\left(x_{0} \mid \mathbf{x}, y_{0}\right) \propto p\left(\mathbf{x} \mid x_{0}, y_{0}\right) p\left(x_{0} \mid y_{0}\right)=p\left(\mathbf{x} \mid x_{0}, y_{0}\right) p\left(x_{0}\right),
$$

where we have assumed that the prior on $x_{0}$ is independent of $y_{0}$. Proportionality constants are always determined by normalization - the requirement that the probability that some event occurs is unity. If we suppose we have no prior information about the $x_{0}$ location of the source, then for the prior $p\left(x_{0}\right)$ we should use a constant over whatever sized region is required. Such a prior is noncommittal about the location of the source. Assume that the photons are emitted independently. Each measured $x_{i}$ follows the likelihood, Eq. (1), and the assumption of independence simply means that $p\left(\mathbf{x} \mid x_{0}\right)$ is the product of the single measurement likelihoods $p\left(x_{i} \mid x_{0}\right)$. Using Bayes law, Eq. (2), and the uniform prior assumption, the full posterior probability is

$$
p\left(x_{0} \mid \mathbf{x}, y_{0}\right) \propto p\left(\mathbf{x} \mid x_{0}, y_{0}\right)=\prod_{i=1}^{N} p\left(x_{i} \mid x_{0}, y_{0}\right) \propto \prod_{i=1}^{N}\left[\frac{y_{0}}{y_{0}^{2}+\left(x_{0}-x_{i}\right)^{2}}\right] .
$$

Again, all proportionality constants are determined by normalization. Here this is the requirement that the integral of $p\left(x_{0} \mid \mathbf{x}, y\right)$ over $x_{0}$ is unity. From here on, we will often drop explicit mention of $y_{0}$ and write the posterior as $p\left(x_{0} \mid \mathbf{x}\right)$.

In the above derivation the posterior probability is proportional to the likelihood because the prior is assumed to be a constant. The likelihood expresses the probability of obtaining the specific set of measurements, given a particular $x_{0}$. We emphasize that Bayes's law is necessary to gain information about $x_{0}$ from the likelihood [2].

If it were known that the source position was limited to a specific region, an appropriate prior would consist of a function that is a positive constant inside the region and zero outside. This prior would have the effect of eliminating the tails of the posterior probability in (2) outside the legitimate region. This prior would alleviate any problem that might exist with the normalization of the prior.

\subsection{Monte Carlo simulation}

To numerically test how well various estimators of $x_{0}$ perform, we need to generate measurements that simulate a series of experiments. The cumulative probably (also called the distribution function), the probability of a measurement $x_{i}<u$, is given by

$$
P\left(x_{i}<u\right)=\int_{-\infty}^{u} p\left(x \mid x_{0}, y_{0}\right) d x=\frac{1}{\pi} \tan ^{-1}\left(\frac{u-x_{0}}{y_{0}}\right)+\frac{1}{2} .
$$

To generate measurements from the Cauchy distribution, one uses a pseudorandom number generator that provides a number $r_{i}$ in the interval $(0,1)$ and then maps the result into the $x_{i}$ value using the inverse of $(4), x_{i}=x_{0}+y_{0} \tan \left[\pi\left(r_{i}-\frac{1}{2}\right)\right]$.

Note that when $u-x_{0} \gg y_{0}, P\left(x_{i} \geq u\right)=1-P\left(x_{i}<u\right) \approx y_{0} / \pi\left(u-x_{0}\right)$. The probability of getting an $x_{i}$ value that is greater than 1000 times the FWHM of the distribution $\left(2 y_{0}\right)$ is 

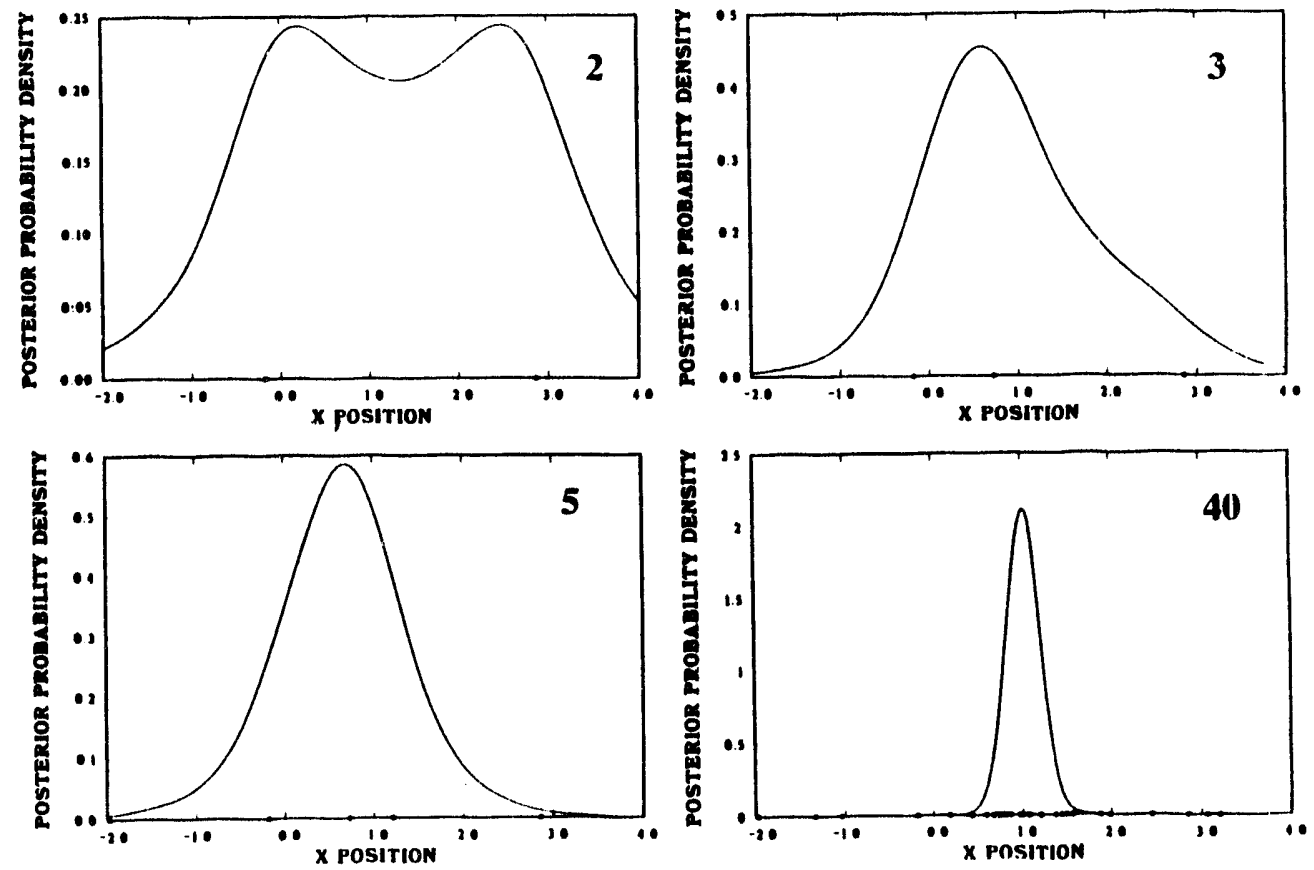

Figure 1: The posterior probability density function for the $x_{0}$ position of the radioactive source assuming that the correct $y_{0}$ is known. Each of these plots is shown for one simulated experiment; the measured $x_{i}$ are displayed on the horizontal axis. The number of measurements is noted in the upper-right corner of each plot.

roughly $1 / 1000$. Thus the Cauchy distribution offers a superb example of a data distribution with outliers.

The posterior probability given by Eq. (3) is plotted in Fig. 1 for specific measurements generated using the Monte Carlo technique described above. The plot for two measurements is bimodal, making it ambiguous to use the maximum posterior probability (see Sect. 3.2) to estimate $x_{0}$. As the number of measurements increases, the width of the posterior density function decreases, indicating less uncertainty in the knowledge of $x_{0}$. The broad tail of the Cauchy likelihood is increasingly suppressed as the number of measurements increases because the posterior probability involves a product of likelihoods of the individual measurements.

\section{Estimation of Location}

\subsection{Mean and median of the measurements}

The average of the $x_{i}$ measurements (or samples) is often used to estimate the central position of their distribution: 


$$
\hat{x}_{0(\text { samp mean })}=\frac{1}{N} \sum_{i=1}^{N} x_{i} .
$$

The variance of the average of $N$ samples taken randomly and independently from an arbitrary density function is easily shown to be $N^{-1}$ times the variance of the original density function, provided such variance exists. Curiously, the density function for the average of $N$ samples from the Cauchy distribution is identical to that for one sample. Because the variance of the Cauchy density function is infinite, so will be the variance of the average of any finite number of samples. However, for a Gaussian density function, this estimator would be the sufficient statistic for the central position and would be optimal in many ways.

An alternative estimator of the center of a sampled distribution is the sample median $\hat{x}_{0 \text { (samp med) }}$, which is supposed to be robust against outliers $[3, \mathrm{pp} .232]$. For odd $N$, the median is defined as the $\frac{1}{2}(N+1)$ th sample in the list of magnitude-ordered measurements; for even $N$, it is defined as the average of the $(N / 2)$ th and the $(N / 2+1)$ th samples from such a list.

\subsection{Bayesian estimators}

The Bayesian viewpoint is that the posterior probability density function for $x_{0}$ summarizes our state of knowledge of $x_{0}$ in probabilistic terms. Various types of estimators can be formed from the posterior. The choice of estimator can be based on how the cost of making an error in the estimated quantity depends on the size of the error [1]. The most commonly used estimator in Bayesian analysis is the $x_{0}$ value at the maximum of the posterior probability, which we designate by $\hat{x}_{0 \text { (MAP) }}$, because it is usually called the maximum a posteriori estimator. The MAP estimator minimizes a cost function that is zero for no error and a positive constant for any finite error.

The estimate $\hat{x}_{0}$ that minimizes the expected mean-square error, i.e. $\int\left(\hat{x}_{0}-x_{0}\right)^{2} p\left(x_{0} \mid \mathbf{x}\right) d x_{0}$, is the mean of the posterior density function:

$$
\hat{x}_{0(\text { post mean })}=\int x_{0} p\left(x_{0} \mid \mathbf{x}\right) d x_{0} .
$$

Defining an integral that is proportional to the $k$ th moment of the posterior given in Eq. (3)

$$
I_{k}(\mathbf{x})=\frac{y_{0}}{\pi} \int_{-\infty}^{+\infty} x_{0}^{k} \prod_{i=1}^{N} \frac{1}{\left[y_{0}^{2}+\left(x_{i}-x_{0}\right)^{2}\right]} d x_{0},
$$

the mean (or first moment ${ }^{1}$ ) of the posterior is

$$
\hat{x}_{0(\text { post mean })}=\frac{I_{1}(\mathbf{x})}{I_{0}(\mathbf{x})} .
$$

The integrand in Eq. (7) has simple $\mathrm{e}^{2}$ poles at $x_{i}^{ \pm} \equiv x_{i} \pm \imath y_{0}$. By interpreting the integral as one along the real axis in the complex plane and closing the contour at $\infty$ in the upper half plane (which contributes nothing provided the integrand falls off faster than $x^{-1}$ ), the desired result is found using the Cauchy residue theorem

\footnotetext{
${ }^{1}$ The $k$ th moment of the posterior is $I_{k}(\mathbf{x}) / I_{0}(\mathbf{x})$.

${ }^{2}$ The procedure described here must be trivially modified when $x_{i}=x_{j}$ for $i \neq j$. However, we need not consider such coincident measurements because they represent a set of zero probability.
} 


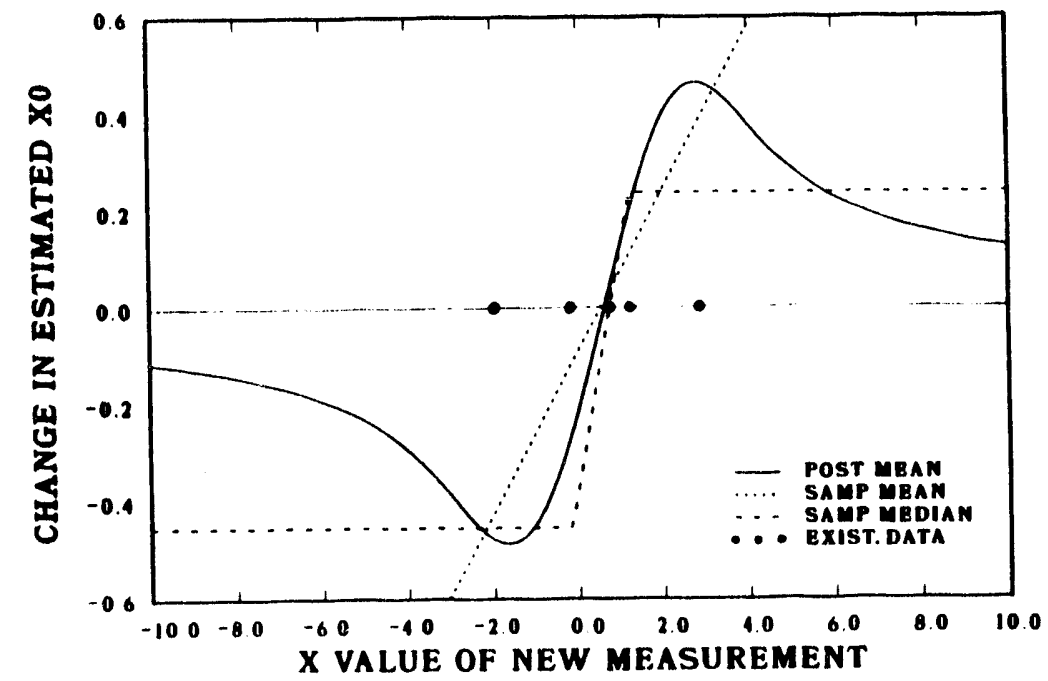

Figure 2: The change in the estimated position of the Cauchy distribution caused by adding a new measurement $x_{6}$ to five existing measurements as a function of the value of the new measurement. The results are shown for several kinds of estimators.

$$
\begin{aligned}
I_{k}(\mathbf{x}) & =\sum_{i=1}^{N}\left(x_{i}^{+}\right)^{k} \prod_{j \neq i} \frac{1}{\left(x_{i}^{+}-x_{j}^{+}\right)\left(x_{i}^{+}-x_{j}^{-}\right)}, 0 \leq k<2 N-1 \\
& =\sum_{i=1}^{N}\left(x_{i}^{+}\right)^{k} \prod_{j \neq i}\left[\frac{1}{\left(x_{i}-x_{j}\right)^{2}+4 y_{0}^{2}}\right]\left[1-\frac{2 \imath y_{0}}{x_{i}-x_{j}}\right], 0 \leq k<2 N-1,
\end{aligned}
$$

where the second expression is obtained by simply rearranging the product.

Note that by its definition (7), $I_{k}$ is real for all allowed $k$. In particular for $k=1$, the factor $\left(x_{i}^{+}\right)^{k}=x_{i}+\imath y_{0}$ in Eq. (10) contributes to two summations, one summation with factor $x_{i}$ and the other summation with factor $\imath y_{0}$. The summation with factor $\imath y_{0}$ is identically $\imath y_{0} I_{0}$. Because $I_{0}$ is real, $\imath y_{0} I_{0}$ is imaginary. Thus the $\imath y_{0}$-tactor summation must be exactly cancelled by the imaginary part of the $x_{i}$-factor summation and we may write

$$
I_{1}(\mathbf{x})=\Re\left\{\sum_{i=1}^{N} x_{i} \prod_{j \neq i}\left[\frac{1}{\left(x_{i}-x_{j}\right)^{2}+4 y_{0}^{2}}\right]\left[1-\frac{2 \imath y_{0}}{\left(x_{i}-x_{j}\right)}\right]\right\}, 0 \leq k<2 N-1 .
$$

Therefore, the posterior mean estimator (8) has the form of a weighted average of the $x_{i}$, $\hat{x}_{0 \text { (post mean) }}=\sum w_{i} x_{i}$, where the sum of the weights is unity. Although this expression looks like a simple variation on the sample average (5), the weights possess a very complicated behaviour. The net effect of the first factor in the product in (11) leads to a diminished contribution from an outlier. But it is very difficult to conceptually grasp the effect of the second factor owing to its complex nature.

Figure 2 shows the behavior of the various estimators when a new measurement is combined with five existing measurements. As the value of the new measurement moves away from the other measurements, its net effect on $\hat{x}_{0 \text { (post mean) }}$ goes to zero. Thus the 
estimator minimizes the contribution of any measurement that lies far from a cluster of other measurements, which seems to be an ideal treatment of outliers. Because the posterior is independent of the order of the measurements, the same behaviour is expected for any measurement. The posterior maximum estimator behaves similarly to the posterior mean. A new measurement affects the sample mean in a linear fashion because it is just a linear combination of all measurements. The outlier sample can drastically affect the sample mean. The sample median behaves quite differently. The change in the median remains constant as long as the $(N+1)$ th sample lies outside the central-most two or three samples, depending on whether $N$ is even or odd, respectively. The estimators based on the posterior are the only ones for which the effect of a single disparate measurement decreases as its descrepancy from the others increases.

The variance of the posterior density function of $x_{0}$ for a particular data vector $\mathbf{x}$ is

$$
\operatorname{var}\left\{p\left(x_{0} \mid \mathbf{x}\right)\right\}=\int\left[x_{0}-\hat{x}_{0 \text { (post mean) })}\right]^{2} p\left(x_{0} \mid \mathbf{x}\right) d x_{0}=\frac{I_{2}(\mathbf{x})}{I_{0}(\mathbf{x})}-\left[\frac{I_{1}(\mathbf{x})}{I_{0}(\mathbf{x})}\right]^{2}, N \geq 2 .
$$

An interesting property of the posterior probability is that its shape depends on $\mathbf{x}$ and is hence usually different for different experiments. See Sect. 4 for its relationship to rms error for $\hat{x}_{0 \text { (post mean) }}$.

\subsection{Fisher-Cramér-Rao lower bound and Fisher information}

The Fisher-Cramér-Rao bound ${ }^{3}$ places a lower bound on the variance of any unbiased estimator $\hat{x}(\mathbf{x}), \operatorname{var}(\hat{x}) \geq \mathcal{I}_{N}^{-1}$, where $\mathcal{I}_{N}$ is the Fisher information

$$
\mathcal{I}_{N} \equiv \int \frac{\partial^{2} \log \left[p\left(x_{0} \mid \mathbf{x}\right)\right]}{\partial x_{0}^{2}} p\left(x_{0} \mid \mathbf{x}\right) d \mathbf{x},
$$

and where $N$, the number of measurements, is the dimension of $\mathbf{x}$. Because the posterior (3) factors, we have $\mathcal{I}_{N}=N \mathcal{I}_{1}$, where $\mathcal{I}_{1}$ is the single-sample Fisher information given by

$$
\mathcal{I}_{1}=\frac{4 y_{0}}{\pi} \int_{-\infty}^{+\infty} \frac{\left(x-x_{0}\right)^{2}}{\left[y_{0}^{2}+\left(x-x_{0}\right)^{2}\right]^{3}} d x=\frac{1}{2 y_{0}^{2}} .
$$

In the last step the integral is evaluated by applying the Cauchy residue theorem (as in Sect. 3.2). Thus the Fisher-Cramér-Rao lower bound on the variance of any unbiased estimator of $x_{0}$ is

$$
\operatorname{var}(\hat{x}) \geq\left[N \mathcal{I}_{1}\right]^{-1}=\frac{2 y_{0}^{2}}{N} \text {. }
$$

It is important to note that this lower bound is valid only for unbiased estimators, i.e. when averaged over all possible data, it yields the correct result $\int \hat{x}_{0}(\mathbf{x}) p\left(\mathbf{x} \mid x_{0}\right) d \mathbf{x}=x_{0}$. We have established that both the sample median and posterior mean are unbiased, that their biases exist for $N \geq 3$, while their variances exist for $N \geq 4$.

\section{Simulation Results}

The performance of the above estimators for $x_{0}$ is tested by simulating $10^{5}$ experiments, each involving a fixed number of measurements $N$, which are independently drawn from a Cauchy distribution as indicated in Sect. 2.2. The parameters are held fixed at $x_{0}=1$ and $y_{0}=1$ throughout. The results are summarized in Table 1 . In these numerical experiments, except for the sample mean, the bias is always observed to be consistent with zero to within its statistical uncertainty, i.e. on the order of the [rms error of the estimator] $/ \sqrt{T}$, where $T$ is the number of trials, or experiments. We observe that the average value of the measurements

\footnotetext{
${ }^{3}$ Fisher stated this lower bound many years before Cramér and Rao $[4$, p. 66].
} 
Table 1: Summary of the performance of several estimators of the central position of a Cauchy distribution observed in $10^{5}$ trials for a fixed number of samples per trial $N$. The estimators used are the mean and the median of the samples, and the maximum and mean of the posterior probability density function. The last two columns give the Fisher-CramérRao lower bound on the rms error and the rms width of the posterior probability.

\begin{tabular}{|c|c|c|c|c|c|c|}
\hline & \multicolumn{5}{|c|}{ rms error in estimated position } & \multirow{2}{*}{ ( } \\
\cline { 2 - 6 } & samp mean & samp median & post max & post mean & FCR & rms post \\
\hline 1 & $2.85 \times 10^{10}$ & $2.85 \times 10^{10}$ & $2.85 \times 10^{10}$ & $2.85 \times 10^{10}$ & 1.414 & $\infty$ \\
2 & $1.43 \times 10^{10}$ & $1.43 \times 10^{10}$ & -- & $1.43 \times 10^{10}$ & 1.000 & $1.43 \times 10^{10}$ \\
3 & $9.52 \times 10^{9}$ & 2.828 & 2.825 & 2.768 & 0.816 & 2.616 \\
5 & $5.71 \times 10^{9}$ & 1.103 & 1.070 & 0.958 & 0.632 & 0.963 \\
10 & $2.86 \times 10^{9}$ & 0.578 & 0.538 & 0.522 & 0.447 & 0.523 \\
20 & $1.43 \times 10^{9}$ & 0.373 & 0.341 & 0.339 & 0.316 & 0.339 \\
40 & $7.14 \times 10^{8}$ & 0.256 & 0.236 & 0.232 & 0.224 & 0.232 \\
\hline
\end{tabular}

performs terribly! This poor performance was anticipated, owing to the infinite variance of the Cauchy distribution. The only reason that the rms error in $\hat{x}_{0 \text { (samp mean) }}$ is not infinite, as mentioned, is that only a finite number of trials are included. The largest $x_{i}$ in the particular sequence of pseudorandom numbers used to generate the $4 \times 10^{6}$ measurements for the $N=40$ test is $9.03 \times 10^{12}$. Because of the symmetry of the likelihood (1) for one and two measurements, all the estimators are identical for $N=1$ and 2 . The posterior mean and maximum perform much better than the sample average for three or more measurements.

The estimators based on the sample median and the maximum of the posterior probability density function perform only slightly worse than the one based on the posterior mean. Just as they demonstrate the weakness of the sample mean estimator, these results underscore the value of the sample median as a simple estimator that is robust against outliers. Empirically, $r m s\left(\hat{x}_{0(\text { samp med })}\right)>r m s\left(\hat{x}_{0(\text { post } \max )}\right)>r m s\left(\hat{x}_{0(\text { post mean })}\right)$, where $r m s$ indicates the rms error from Table 1.

The Fisher-Cramér-Rao lower bound on the rms error is seen to be a valid lower bound for the estimators summarized in the table, which only begin to approach the lower bound for $N \geq 20$.

It is natural to ask whether the posterior is predictive of the uncertainty in an estimator. The rms width of the posterior for our Cauchy problem $r m s\left\{p\left(x_{0} \mid \mathbf{x}\right)\right\}$ may be calculated by taking the square root of the variance in $x_{0}$, calculated using Eq. (12). The results for the simulated experiments, shown in the last column of the table, indicate that this calculation does predict the rms error in the $\hat{x}_{0 \text { (post mean) }}$ estimator.

We note that the shape of the posterior depends on the measured data, as inferred from Fig. 1. This behavior is different for a Gaussian likelihood with uniform prior, for which the width and shape of the posterior for a fixed number of data samples does not depend on the actual data values. We find in $10^{5}$ trials for $N=5$ that when the trials are selected on basis of $\operatorname{rms}\left\{p\left(x_{0} \mid \mathbf{x}\right)\right\}$, the rms error in the estimator $\hat{x}_{0(\text { post max) }}$ for those trials closely reproduces the chosen $r m s\left\{p\left(x_{0} \mid \mathbf{x}\right)\right\}$. This result indicates that the posterior probability density function derived for each experiment provides information about the certainy of inferences made on the basis of that experiment. It is intriguing to consider how such 
information might be used to make decisions about whether more data should be taken to achieve a desired accuracy of interpretation.

\section{Discussion}

The prior used in the Bayesian analysis was the uniform prior. Because the uniform prior on the real number line is not normalizable, this analysis must be viewed as a limit over normalized priors [5]. In practice, the prior should reflect the state of prior knowledge.

The Bayesian analysis also assumed that the measurement interval was the complete $x$ axis. When the measurement interval is finite, and assuming that a fixed number of measurements are made, the posterior has asymptotically nonzero constant tails. The reason for this is that the probability of the measurements is then simply the product of the probabilities $p\left(x_{i} \mid x_{0}, y_{0}\right)$ of Eq. (1), with each normalized to unity over the finite measurement inteval. For large $x_{0}$ the normalization constant is effectively the width of the measurement interval times the value of the Cauchy distribution tail in that interval (Eq. (2) may be used to establish the precise relationship). Thus, the normalization constant has the same large $x_{0}$ behavior as the Cauchy distribution that it normalizes, and this gives rise to the asymptotically nonzero constant tail.

For a source of fixed intensity the assumption of fixing the number of measurements corresponds to varying the time interval that the measurements are taken within, measuring until the specified number of photons is gathered. A more reasonable assumption is that the time interval that measurements are taken within is fixed. When this is done the intensity of the source must be taken into account, which translates into a different likelihood for the measurements. In particular, the number of measurements follows a Poisson distribution, so that the likelihood for $N$ measurements discussed in the last paragraph is modified by the factor $P\left(N \mid x_{0}, y_{0}\right)=e^{-\lambda} \lambda^{N} / N$ ! where $\lambda=\lambda\left(x_{0}, y_{0}\right)$ is the source intensity times the total probability that a measurement will occur in the measurement interval.

\section{Acknowledgments}

We acknowledge many helpful discussions with Gregory S. Cunningham who suggested using the sample-median estimator. This work was supported by the United States Department of Energy under contract number W-7405-ENG-36.

\section{References}

[1] K. M. Hanson. Introduction to Bayesian image analysis. Proc. SPIE, vol. 1898:716-731, 1993.

[2] S. F. Gull. Bayesian inductive inference and maximum entropy. In G. J. Erickson and C. R. Smith, editors, Maximum Entropy and Bayesian Methods in Science and Engineering (Vol. 1), pages 53-74. Kluwer Academic, 1989.

[3] J. L. Devore. Probability and Statistics for Engineering and the Sciences. Brooks/Cole, Monterey, 1987.

[4] H. L. Van Trees. Detection, Estimation, and Modulation Theory - Part I. John Wiley and Sons, New York, 1968. 
[5] G. E. P. Box and G. C. Tiao. Inference in Statistical Analysis. John Wiley and Sons, New York, 1973 (reprinted 1992). 

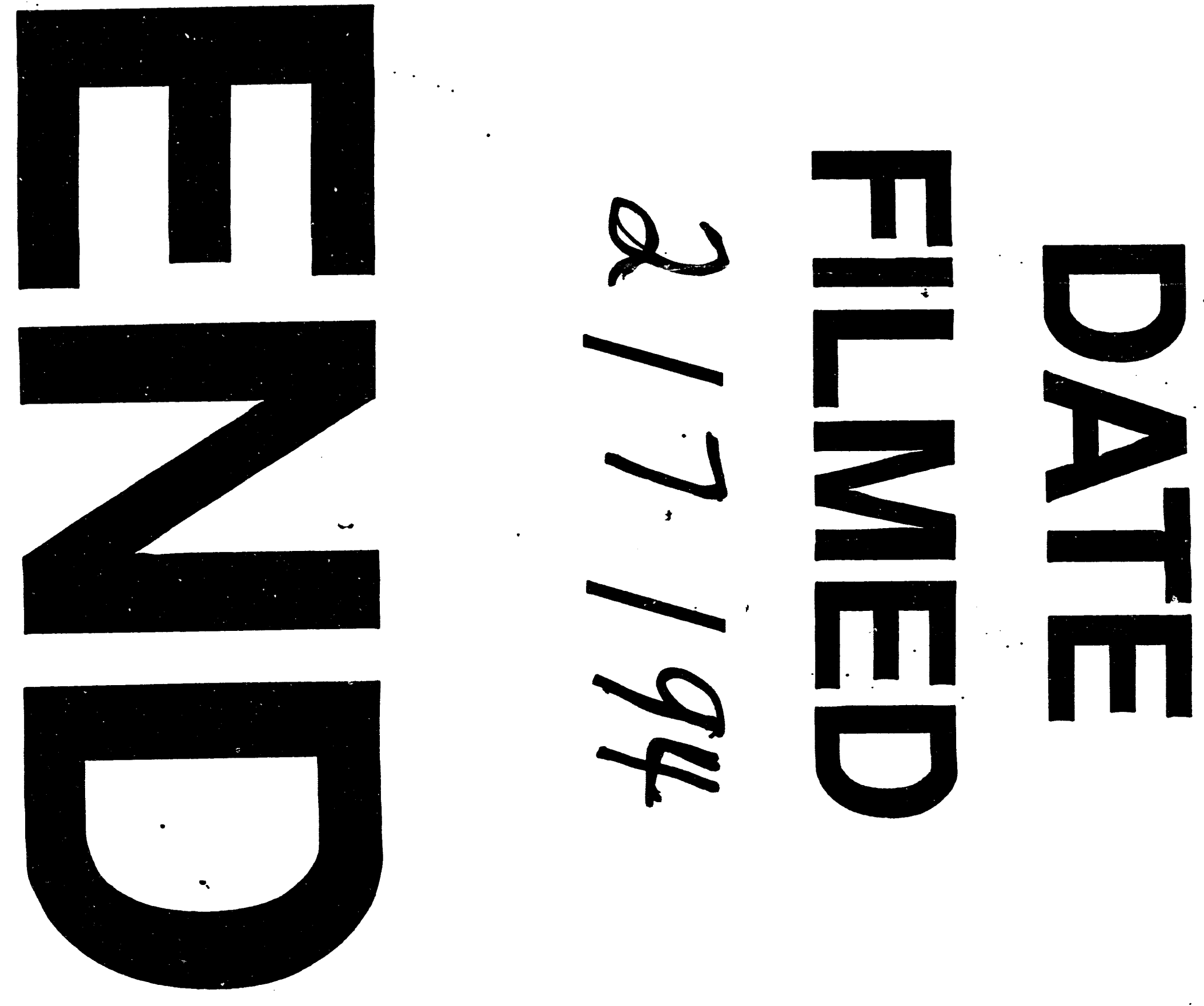